\title{
Delayed Pseudoaneurysm Rupture and Management After Covered Stent Placement for Post Pancreaticoduodenectomy Hemorrhage: A
}

\section{Case Report}

\author{
Ho-Hsian Yen, ${ }^{1,2}$ Chien-An Liu, ${ }^{1,2,}{ }^{*}$ Hsiou-Shan Tseng, ${ }^{1,2}$ and I-Ming Chen ${ }^{3,2}$ \\ ${ }^{1}$ Department of Radiology, Taipei Veterans General Hospital, Taipei City, Taiwan \\ ${ }^{2}$ School of Medicine, National Yang-Ming University, Taipei, Taiwan \\ ${ }^{3}$ Division of Cardiovascular Surgery, Department of Surgery, Taipei Veterans General Hospital, Taipei City, Taiwan \\ "Corresponding author: Chien-An Liu, Department of Radiology, Taipei Veterans General Hospital, No.201, Sec.2, Shih-Pai Rd, Taipei, 11217, Taiwan. Tel: +886-228712121, Fax: \\ +886-228732131, E-mail: caliu@vghtpe.gov.tw
}

Received 2016 October 04; Revised 2017 January 23; Accepted 2017 March 08.

\begin{abstract}
The development of a pseudoaneurysm following Fluency ${ }^{\circledR}$ Plus-covered stent placement for post-Whipple operation bleeding has not been reported. A 79 year-old man diagnosed with pancreas head cancer received Whipple operation and suffered from subsequent gastroduodenal artery stump bleeding. The hemorrhage was treated successfully by covered stent placement at the common hepatic artery. However, two episodes of pseudoaneurysm formation at the proximal stent edge occurred successively in the followup period and were treated with endovascular managements. Sequential development of pseudoaneurysm after covered stent placement for post-Whipple operation bleeding has not been reported in available literature. We consider the pseudoaneurysm formation is not only a consequence of visceral inflammation, but also related to the properties of a stent like rigidity in this case.
\end{abstract}

Keywords: Covered Stent Placement, Endovascular Management, Post-Pancreaticoduodenectomy Hemorrhage, Pseudoaneurysm

\section{Introduction}

Mortality after pancreaticoduodenectomy (PD) has decreased significantly in recent years. However, post-PD bleeding, or pseudoaneurysm formation, remains the most life-threatening complication $(1,2)$. Several studies have described endovascular treatment with covered stent placement for the bleeding event (3-8). However, a literature review showed that the development of a pseudoaneurysm following Fluency ${ }^{\circledR}$ Plus-covered stent placement for post-Whipple operation bleeding has not been reported. Here, we report a case of a pseudoaneurysm that developed in the proximal edge of a Fluency ${ }^{\circledR}$ Plus-covered stent within the follow-up period after covered-stent deployment for post-PD bleeding. The patient was treated with further endovascular management.

\section{Case Presentation}

A 79-year-old man suffered from tea-colored urine, and fatigue for 1 month before being admitted to our hospital. Endoscopic retrograde cholangiopancreatography revealed a bulging papilla with superficial ulceration. Biopsy of the distal common bile duct revealed adenocarcinoma.
A modified Whipple operation with limited antrectomy was performed after a preoperation survey. On postoperative day 23, hypotension and a hemoglobin drop (11.3 to > $9.5 \mathrm{mg} / \mathrm{dL}$ ) were detected. Emergency computed tomography (CT) scan showed small out-pouching with clip migration at the gastroduodenal artery (GDA) stump. Thus, after measuring the diameter of the common hepatic artery (CHA) in pre-operative image and determining the stent graft size, angiography was performed with Seldinger technique for right common femoral artery (CFA) cannulation. Under over-the-wire-based catheter technique, a $10 \times 60$ mm Fluency ${ }^{\circledR}$ Plus-covered stent (Bard Peripheral Vascular, Tempe, AZ) was deployed in the CHA to cover the GDA stump (Figure 1A-C). Thereafter, he was discharged from the hospital in a stable condition.

After a 6-month follow-up, the patient visited our emergency department, complaining of abdominal discomfort. CT scan findings revealed a 5-cm proximal common hepatic artery pseudoaneurysm with contrast extravasation, and a relatively straight shape of the Fluency ${ }^{\circledR}$ Plus-covered stent (Figure 2A-B). Emergency angiography showed a large pseudoaneurysm arising from the proximal edge of the covered stent. Under the same technique mentioned 

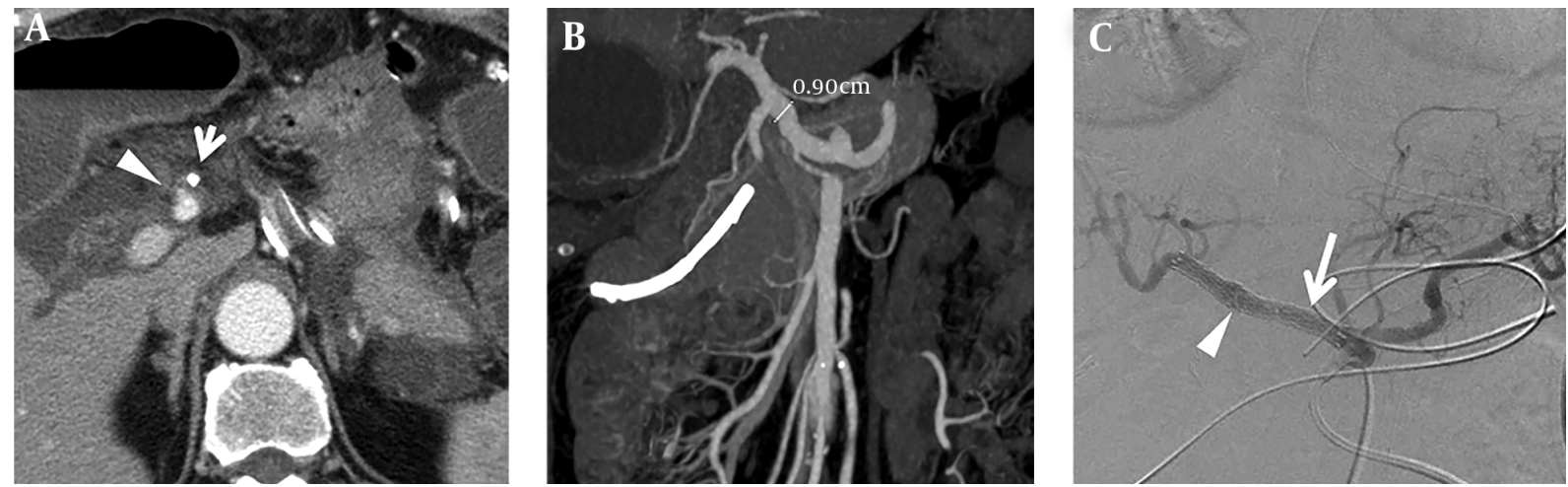

Figure 1. A 79- year-old man suffered from gastroduodenal artery (GDA) stump bleeding at postoperative day 23 and was treated with covered stent placement. A, CT scan showed small arterial enhancing out-pouching (arrowhead) at the GDA stump and clip migration (arrow) with fluid accumulation around the artery. B, Stent graft size was determined after measuring the common hepatic artery (CHA) diameter about $9 \mathrm{~mm}$ in size from the CT image performed before pancreaticoduodenectomy. C, One Fluency ${ }^{\circledR}$ Plus-covered stent (arrow) was deployed to cover the GDA stump (arrowhead) which is the same location in CT image (A).

above, the splenic artery was first embolized to prevent an endoleak; a $10 \times 60 \mathrm{~mm}$ Fluency ${ }^{\circledR}$ Plus-covered stent (Bard Peripheral Vascular, Tempe, AZ) was then deployed from the orifice of the celiac trunk to the distal end of the hepatic proper artery (Figure 2C-D).

Two months after being discharged, the patient continued to suffer from back pain. CT scan detected development of a pseudoaneurysm at the proximal edge of the celiac-covered stent, with hematoma and fluid accumulation along the celiac axis (Figure 3A). Additionally, a mycotic pseudoaneurysm was diagnosed on the basis of the initial clinical presentation, leukocytosis (WBC: 22500/cumm), imaging findings of soft tissue density and perivascular fluid collection around the vessel, and organisms from blood cultures. To solve this problem, the cardiovascular surgeon preferred endovascular management to conventional open surgery because of the high mortality rate in this condition. In the diagnostic angiography image, the ruptured pseudoaneurysm was located superior to the junction between the celiac root and abdominal aorta (Figure 3B-C). Celiac trunk coil embolization cannot seal the pseudoaneurysm. Therefore, the surgeon decided to perform chimney technique combined with coil embolization to prevent potential reflux or endoleak from the celiac trunk. After puncturing both the left brachial artery and left CFA, celiac coil embolization was performed first. Then, we cannulated the wire into the superior mesenteric artery (SMA) via the left brachial artery sheath and deployed a Gore ${ }^{\circledR}$ Viabahn ${ }^{\circledR} 8 \mathrm{~mm} \times 10 \mathrm{~cm}$ stent (W.L. Gore, Flagstaff, AZ) in the SMA for chimney technique. A 34 $\mathrm{mm} \times 77 \mathrm{~mm}$ Zenith ${ }^{\circledR} \mathrm{TX}{ }^{\circledR}{ }^{\circledR}$ TAA TBE-34-77 stent graft (Cook Inc., Bloomington, Indiana) was deployed with coverage of celiac trunk in the abdominal aorta via left CFA. Another Zilver $^{\circledR} 8 \mathrm{~mm} \times 8 \mathrm{~cm}$ stent (Cook Inc., Bloomington, In- diana) was introduced inside the Gore ${ }^{\circledR}$ Viabahn ${ }^{\circledR}$ in the SMA to prevent compression from the Zenith ${ }^{\circledR} \mathrm{TX} 2{ }^{\circledR}$ stent graft. The immediate follow-up angiogram showed patent abdominal stent graft with obliteration of celiac trunk and no contrast medium extravasation (Figure 3D-F). Following this procedure and combined with an 8-week antibiotic treatment, the patient was discharged in stable condition and received long-term follow-up without further complication in the latest follow-up imaging (Figure 4).

\section{Discussion}

Post-PD hemorrhage is reported to occur in $2 \%-8 \%$ of patients and is life threatening, with a reported mortality rate of $18 \%$ - 47\% (9). Post-PD hemorrhage can be classified as either early or delayed (10). Early post-PD hemorrhage occurs within 24 hours after the end of the operation, and is most often a result of technical failure or insufficient management of underlying coagulopathy. Delayed postPD hemorrhage occurs more than 24 hours after the end of the operation, and is typically caused by pseudoaneurysm rupture as a consequence of visceral inflammation, such as pancreatic leakage adjacent to the arterial wall $(4,5,10)$.

Transarterial embolization (TAE) and covered stent placement (CSP) are reported as approaches for handling post-PD hemorrhage $(4,6)$. TAE uses coils to embolize the target artery and carries the risk of inducing liver infarction or liver abscess formation. CSP is safer than TAE because the pseudoaneurysmal wall may be friable and unable to withstand pressure from endovascular coils, which may cause rupture of the arterial wall and uncontrollable bleeding (4). Before this case, we used to deploy Fluency ${ }^{\circledR}$ Plus-covered stent treating post-PD hemorrhage in our institute. 

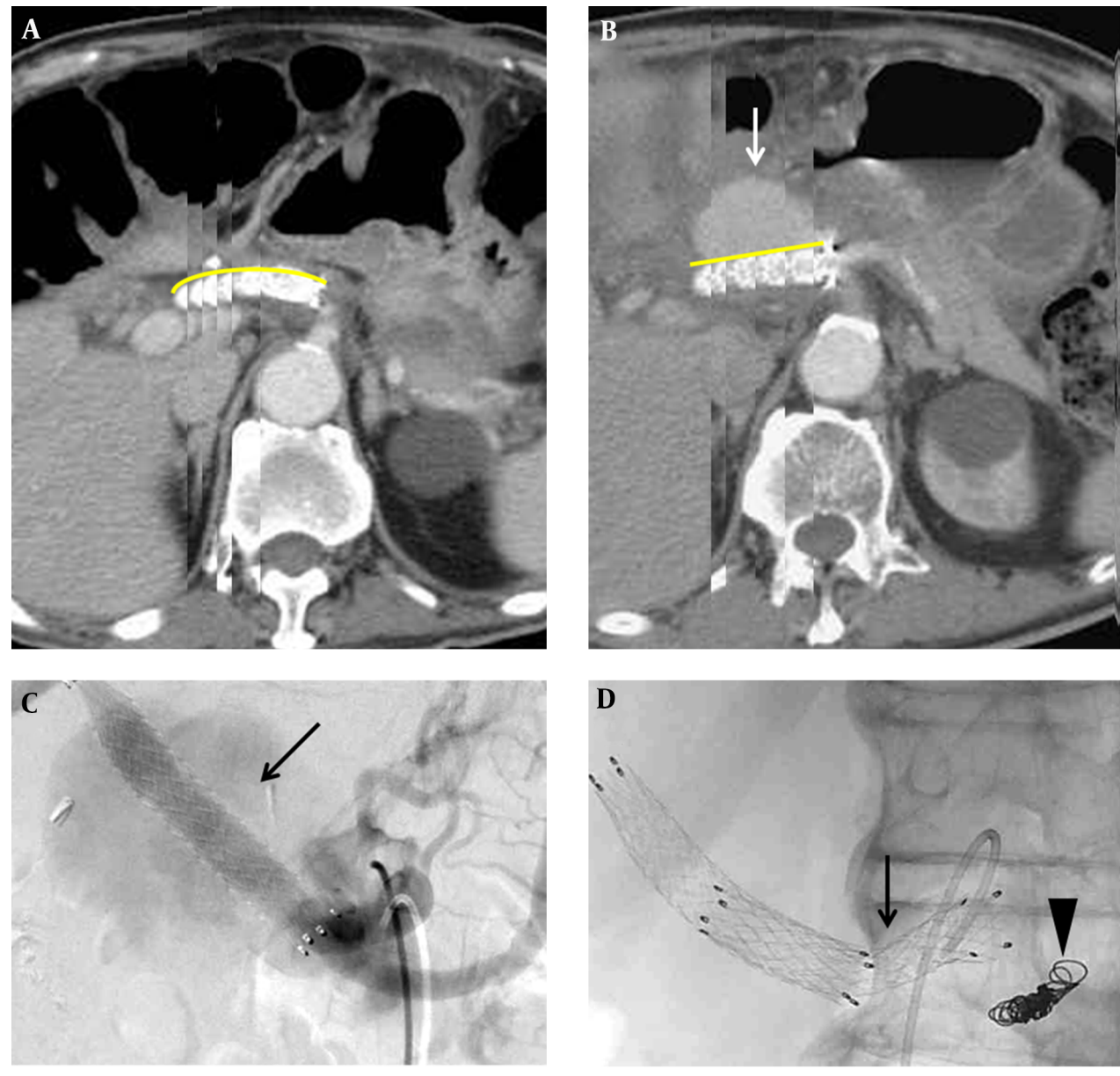

Figure 2. Development of the pseudoaneurysm from the proximal edge of the covered stent after a 6-month follow-up and management. A, In the first 2-month follow-up, the patient was stable after Fluency ${ }^{\circledR}$ Plus-covered stent deployment, serial images recombined in graphics software show a relatively curved shape of the stent (yellowish curve) and no evidence of pseudoaneurysm formation. B, Six months after Fluency ${ }^{\circledR}$ Plus-covered stent deployment, pseudoaneurysm developed (arrow) and a relatively straight shape of the stent (yellowish line) as compared with (A). C, Angiographic imaging showed a large pseudoaneurysm arising from the proximal edge of the covered stent (arrow). D, Embolization of the splenic artery (arrowhead) and another $10 \times 60 \mathrm{~mm}$ Fluency ${ }^{\circledR}$ Plus-covered stent placement for pseudoaneurysm (arrow).

Development of a pseudoaneurysm after stent implantation is rare. It has been described only in the coronary artery and iliac artery (11-13). However, development of a pseudoaneurysm following a Fluency ${ }^{\circledR}$ Plus-covered stent placement for post-Whipple operation bleeding has never been reported. We consider that both the intrinsic properties of the chosen stent and the erosion of vasculature resulting from intraabdominal infection and pancreatic fluid adjacent to the surgical anastomosis may influence pseudoaneurysm formation after covered stent placement. The closed cell structure in the Fluency ${ }^{\circledR}$ Plus stent makes it relatively rigid compared with that of a flexible-covered stent such as the Gore ${ }^{\circledR}$ Viabahn ${ }^{\circledR}$. To our knowledge, pseudoaneurysm formation at the edge of Gore ${ }^{\circledR}$ Viabahn ${ }^{\circledR}$ stent has not been reported in available literature, but there is a reported case with regular hemodialysis receiving a Fluency ${ }^{\circledR}$ Plus stent deployment for transposed brachiobasilic arteriovenous fistula stenosis who experienced a protrusion of the stent strut that penetrated the wall of the fistula 6 months after the procedure (14). The author speculated that the rigid properties of the Fluency ${ }^{\circledR}$ Plus stent graft may have caused the stent to straighten (14). In our case, another Fluency ${ }^{\circledR}$ Pluscovered stent was deployed for the first episode of pseudoaneurysm formation because of the emergent condition and only Fluency ${ }^{\circledR}$ Plus-covered stent was available at that time in our department.

Diagnosis of an infected aneurysm is based on imaging the aneurysm, and infection is confirmed by culturing an organism from the blood. CT imaging findings of an infected aneurysm include a saccular, eccentric, or multilobulated shape, soft tissue inflammation around vessels, and 

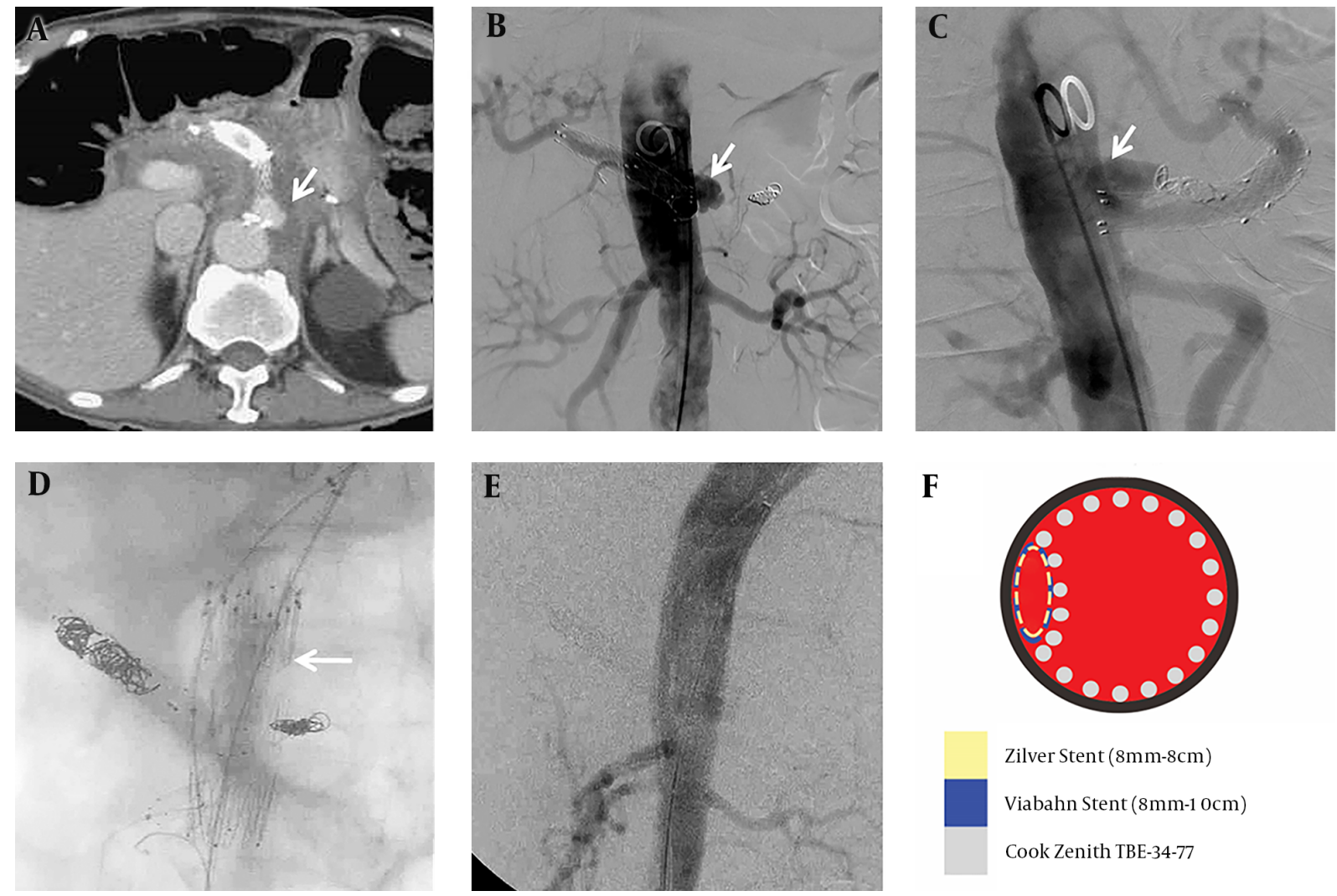

$\mathbf{F}$

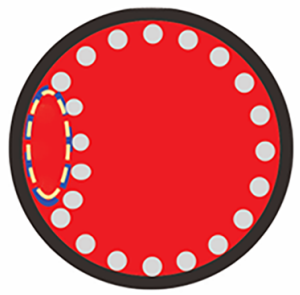

Zilver Stent $(8 \mathrm{~mm}-8 \mathrm{~cm})$

Viabahn Stent $(8 \mathrm{~mm}-10 \mathrm{~cm})$

Cook Zenith TBE-34-77

Figure 3. Recurrent complication of pseudoaneurysm formation from the stent edge two months after the last event and management. A, Recurrent pseudoaneurysm developed (arrow) at the proximal edge of the Fluency ${ }^{\circledR}$ Plus-covered stent. B and C, The anterior-posterior projection and lateral projection in diagnostic angiogram showed an outpouching pseudoaneurysm located on the left side and superior to the junction between celiac root and abdominal aorta (arrow). D, A chimney technique with a Gore ${ }^{\circledR}$ Viabahn ${ }^{\circledR} 8 \mathrm{~mm} \times 10 \mathrm{~cm}$ stent (W.L. Gore, Flagstaff, AZ) in the superior mesenteric artery (SMA), a Zenith ${ }^{\circledR}$ TX2 ${ }^{\circledR}$ TAA TBE-34-77 stent graft (Cook Inc., Bloomington, Indiana) in the abdominal aorta (arrow), and a Zilver ${ }^{\circledR} 8 \mathrm{~mm} \times 8 \mathrm{~cm}$ stent (Cook Inc., Bloomington, Indiana) inside the Gore ${ }^{\circledR}$ Viabahn ${ }^{\circledR}$ in the SMA. E, No contrast medium extravasation or residual pseudoaneurysm was detected in the immediate follow-up angiogram. F, Illustration of the endovascular management in axial view with different colors as different implantations.

perivascular fluid collection. In our case, mycotic pseudoaneurysm was diagnosed at the second episode by clinical symptoms, laboratory findings and imaging.

Conventional surgical resection, extensive local debridement, and revascularization through in situ reconstruction or extraanatomic bypass are the gold standard treatments for mycotic aneurysm, but they carry a high rate of in-hospital mortality about $22.7 \%$ and thirty-day mortality of $18.2 \%$ (15). The endovascular aneurysm repair (EVAR) is less invasive compared with conventional open repair and reduces mortality and morbidity rates $(16,17)$. The European multicenter study on endovascular treatment of mycotic aortic aneurysms from the American Heart Association Circulation Journal included the largest population sample to date intending to assess the durability of endovascular treatment. The study concluded that endovascular treatment is feasible and durable for most patients, especially in surgical high-risk cases; however, late-infection related complications occur because the infected tissue is not resected. Thus, long-term antibiotic treatments and follow-ups are warranted. Under the circumstances, EVAR could be considered a palliative option or a bridge to later elective open repair (16).

In conclusion, development of a pseudoaneurysm following Fluency ${ }^{\circledR}$-covered stent placement for post-PD bleeding because of intrinsic properties of the chosen stent has not been reported. A flexible-covered stent such as the Gore ${ }^{\circledR}$ Viabahn ${ }^{\circledR}$ may be an effective option for resolving this problem. And endovascular treatment is feasible for mycotic pseudoaneurysm, especially in surgical high-risk cases. 


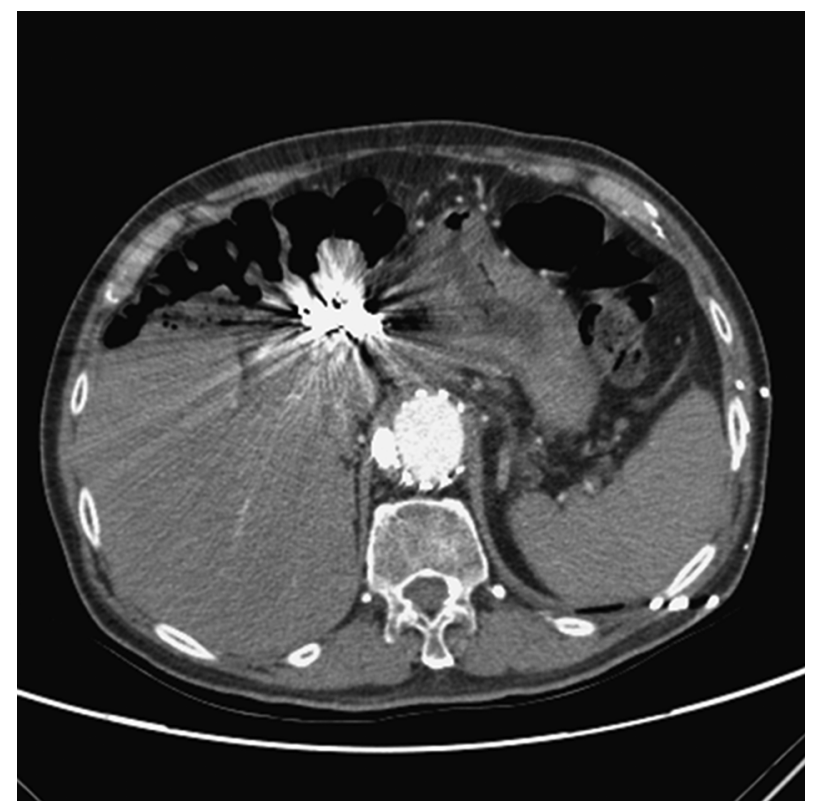

Figure 4. The latest follow up image showed the post endovascular management status without further complication

\section{Acknowledgments}

No.

\section{Footnotes}

Authors' Contributions: Study concept and design, HoHsian Yen, Chien-An Liu; acquisition of data, Chien-An Liu, I-Ming Chen; analysis and interpretation of data, Ho-Hsian Yen, Chien-An Liu, and Hsiou-Shan Tseng; drafting of the manuscript, Ho-Hsian Yen, and Chien-An Liu; critical revision of the manuscript for important intellectual content, Chien-An Liu.

Financial Disclosure: All authors have no financial interests related to the material in the manuscript.

\section{Funding/Support: No.}

\section{References}

1. Wei HK, Wang SE, Shyr YM, Tseng HS, Tsai WC, Chen TH, et al. Risk factors for post-pancreaticoduodenectomy bleeding and finding an innovative approach to treatment. Dig Surg. 2009;26(4):297-305. doi: 10.1159/000228245. [PubMed: 19602889].

2. Feng J, Chen YL, Dong JH, Chen MY, Cai SW, Huang ZQ. Postpancreaticoduodenectomy hemorrhage: risk factors, managements and outcomes. Hepatobiliary Pancreat Dis Int. 2014;13(5):513-22. doi: 10.1016/S1499-3872(14)60276-9. [PubMed: 25308362].
3. Eyheremendy EP, Mendez P, McCormack L, Primo JC, Montano Y, Sierre S. [Endovascular treatment of an hepatic artery pseudoaneurysm following pancreaticoduodenectomy]. Acta Gastroenterol Latinoam. 2015;45(1):80-4. [PubMed: 26076520].

4. Huo Y, Chi J, Zhang J, Liu W, Liu D, Li J, et al. Endovascular intervention for delayed post-pancreaticoduodenectomy hemorrhage: clinical features and outcomes of transcatheter arterial embolization and covered stent placement. Int J Clin Exp Med. 2015;8(5):7457-66. [PubMed: 26221289].

5. Iswanto S, Nussbaum ML. Hepatic artery pseudoaneurysm after surgical treatment for pancreatic cancer: minimally invasive angiographic techniques as the preferred treatment. $N$ Am J Med Sci. 2014;6(6):287-90. doi: 10.4103/1947-2714.134377. [PubMed: 25006566].

6. Lim SJ, Park KB, Hyun DH, Do YS, Park HS, Shin SW, et al. Stent graft placement for postsurgical hemorrhage from the hepatic artery: clinical outcome and CT findings. J Vasc Interv Radiol. 2014;25(10):1539-48. doi:10.1016/j.jvir.2014.06.023. [PubMed: 25149115].

7. Narumi S, Hakamda K, Toyoki Y, Noda H, Sato T, Morohashi H, et al. Endovascular treatment of life-threatening pseudoaneurysm of the hepatic artery after pancreaticoduodenectomy. Hepatogastroenterology. 2007;54(79):2152-4. [PubMed: 18251179].

8. Robinson K, Rajebi MR, Zimmerman N, Zeinati C. Postpancreaticoduodenectomy hemorrhage of unusual origin: treatment with endovascular embolization and the value of preoperative CT angiography. J Radiol Case Rep. 2013;7(4):29-36. doi: 10.3941/jrcr.v7i4.1254. [PubMed: 23705050].

9. Ellison EC. Evidence-based management of hemorrhage after pancreaticoduodenectomy. Am J Surg. 2007;194(1):10-2. doi: 10.1016/j.amjsurg.2006.12.034. [PubMed: 17560901].

10. Wente MN, Veit JA, Bassi C, Dervenis C, Fingerhut A, Gouma DJ, et al. Postpancreatectomy hemorrhage (PPH): an International Study Group of Pancreatic Surgery (ISGPS) definition. Surgery. 2007;142(1):20-5. doi:10.1016/j.surg.2007.02.001. [PubMed:17629996].

11. Caruso M, Evola S, Fattouch K, Bracale UM, Incalcaterra E, La Franca E, et al. Chest pain due to late huge coronary pseudoaneurysm following stent implantation. Intern Med. 2011;50(6):577-9. doi: 10.2169/internalmedicine.50.4566. [PubMed: 21422681].

12. Demir S, Parildar M, Oran I, Memis A. [Stent originating pseudoaneurysm: endovascular treatment of a rare complication with hemobahn-covered stent (case report)]. Tani Girisim Radyol. 2003;9(4):462-5. [PubMed: 14730957].

13. Kishi K, Hiasa Y, Takahashi T. Delayed development of a giant coronary pseudoaneurysm after stent placement for chronic total occlusion. $J$ Invasive Cardiol. 2003;15(5):273-6. [PubMed:12730637].

14. Sequeira A, Artikov S, Abreo K. Stent strut protrusion-an uncommon complication of stent placement in a fistula. Semin Dial. 2014;27(5):529-32. doi:10.1111/sdi.12193. [PubMed: 24438105].

15. Dubois M, Daenens K, Houthoofd S, Peetermans WE, Fourneau I. Treatment of mycotic aneurysms with involvement of the abdominal aorta: single-centre experience in 44 consecutive cases. Eur J Vasc Endovasc Surg. 2010;40(4):450-6. doi: 10.1016/j.ejvs.2010.07.017. [PubMed: 20719550].

16. Sorelius K, Mani K, Bjorck M, Sedivy P, Wahlgren CM, Taylor P, et al. Endovascular treatment of mycotic aortic aneurysms: a European multicenter study. Circulation. 2014;130(24):2136-42. doi: 10.1161/CIRCULATIONAHA.114.009481. [PubMed: 25378548].

17. Kan CD, Lee HL, Yang YJ. Outcome after endovascular stent graft treatment for mycotic aortic aneurysm: a systematic review. J Vasc Surg. 2007;46(5):906-12. doi: 10.1016/j.jvs.2007.07.025. [PubMed: 17905558]. 